\title{
Therapeutic application of adipose-derived stromal vascular fraction in diabetic foot
}

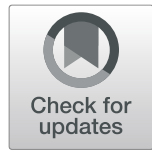

\author{
Xiansheng Zhao', Jiamin Guo ${ }^{2}$, Fangfang Zhang ${ }^{1}$, Jue Zhang ${ }^{3}$, Delin Liu ${ }^{3}$, Wenjun Hu ${ }^{{ }^{*}}$, Han Yin ${ }^{3^{*}}$ and Liang Jin ${ }^{*^{*}}$
}

\begin{abstract}
Diabetic foot is one of the severest complications of diabetes. In severe cases, this disease may be lead to amputation or even death due to secondary infection and ischemic necrosis. Since the ineffectiveness of traditional therapy, autologous stem cell transplantation has been used to treat diabetic foot. This simple, safe, and effective therapy is expected to be applied and promoted in the future.

In this review, we described the detailed pathogenesis of diabetic foot and the common clinical treatments currently used. We also revealed vascular remodeling as the potential mechanism of therapeutic functions of adipose-derived stromal vascular fraction (SVF) in treating diabetic foot.
\end{abstract}

Keywords: Diabetic foot, Stem cells therapy, Stromal vascular fraction (SVF), Cytokine, Vascular reconstruction

\section{Background}

Recently, the incidence of diabetes is increasing in the world, and the age of onset has decreased year by year. Diabetes has become a health problem that cannot be ignored and the prolonged presence of hyperglycemia could induce various complications including diabetic foot [1].

Diabetic foot is caused by diseases of peripheral blood vessels at different degrees and abnormalities of the distal lower limb nerves in diabetic patients, resulting in foot infections, ulcers, and deep tissue damage [2]. Peripheral vascular disease of diabetes often involves the lower extremity arteries. At the early stage, it is mainly characterized by intermittent claudication. As the arterial stenosis increases, the patients could have rest pain or even could not walk. Later, the ischemia with infection and neuropathy could cause local tissue necrosis, ulcers, and gangrene, leading to ischemic diabetic foot

\footnotetext{
*Correspondence: wjhu@cpu.edu.cn; yinhan789@163.com;

ljstemcell@cpu.edu.cn

'School of Life Science and Technology, China Pharmaceutical University, Nanjing 211198, Jiangsu Province, China

${ }^{3}$ Department of Endocrinology, The Affiliated ZhongDa Hospital of Southeast University, Nanjing 210009, Jiangsu Province, China

Full list of author information is available at the end of the article
}

[3]. Finally, $10 \%$ of patients' toes have to be amputated, and the serious infection caused by diabetic foot might even threaten their lives [4]. It has been reported that infection is the main cause of disability and death in diabetic foot patients, while neurogenic and ischemic infections account for $45 \sim 60 \%$, of which $25 \sim 44 \%$ are caused by the neuropathy, and $10 \%$ are caused by bloodlacking [5]. This disease affects the patients' life quality and brings a heavy economic burden to their families.

Since the pathological process of diabetic foot is extremely complicated, traditional medical therapy, vascular bypass, interventional surgery, and amputation treatment methods have certain limitations [6]. Stem cell transplantation technology for the treatment of diabetic foot is a hotspot of transplanted angiogenesis in recent years. Stem cells have the characteristics of high selfrenewal, proliferation, and multiple differentiation potential, as well as paracrine effects, which promote the regeneration of blood vessels and nerve tissues around the transplant site so that the affected ulcers could achieve blood circulation reconstruction and blood supply improvement and, eventually, achieve the purpose of healing diabetic foot $[7-9]$. 
The stromal vascular fraction is an active component in adipose tissue and contains a variety of cells including adipose stem cells [10]. Since the various cell types, they have strong regenerative potentials such as angiogenesis, tissue remodeling, and immune regulation. It has been used for clinic treatment of various conditions and shown good safety and healing effects. Moon and colleagues injected SVF cells into the affected area of diabetic foot patients, and the microvascular blood flow of the wound surface was found significantly increased with no adverse reactions $[11,12]$. Studies found that SVF could secrete a variety of cytokines, differentiate into various cells such as endothelial cells, and have the potential to participate in angiogenesis in vivo. Therefore, it can improve local blood circulation and form the microenvironment that is conducive to tissue cell survival and functional maintenance [13-15].

\section{Pathogenesis of diabetic foot Diabetic foot caused by neuropathy}

The most common complication of diabetes is diabetic neuropathy, accounting for $30 \%$ to $50 \%$ of diabetic patients. The incidence is similar for men and women, but men usually occur earlier than women as their lifestyle and testosterone deficiency [16]. The symptoms of diabetic neuropathy include central neuropathy and peripheral neuropathy, resulting in sensory, motor, and autonomic neuropathy. The pathogenesis of the disease may be related to the increase of neurotrophic factors and the decrease or disappearance of protective factors [17]. Sensory neuropathy is an important cause of diabetic foot. It often involves the small nerves that innervate the pain and temperature of the foot, which leads to a decrease in the sensitivity of the patient to stressrelated trauma and skin damage. Therefore, the selfprotection mechanism is lost, and the harmful stimulation cannot be sensed in time so that the foot is vulnerable to injury [18]. Motor neuropathy mainly affects small peripheral nerves, which lead to abnormal innervation of the gastrocnemius muscle group (mainly flexor) in feet. This imbalance in innervation will cause a change in the shape of the foot, causing the humeral head to protrude. Meanwhile, under the friction of improper footwear and gravity distribution, it is easy to cause foot damage, which leads to foot deformity and ulcer [19]. Autonomic neuropathy is the most complicated in the diabetic neuropathy, which could involve all organs and systems of the whole body. After the occurrence of autonomic neuropathy, the ability of autonomic regulation of skin blood flow is lost. At the same time, the arteriovenous short circuit and shunt increase. Although the skin temperature is not low and the color is pink, the nutritional blood supply has decreased [20]. Also, autonomic neuropathy could cause a lack of secretion of sweat and lipid glands, which makes the skin dry, prone to chapped, and become the gateway to bacterial invasion, eventually leading to ulcers [21].

\section{Diabetic foot caused by vascular lesion}

Diabetes can cause a wide range of vascular diseases, involving arteries, veins, and capillaries. Vascular disease leads to the decrease or even occlusion of blood flow in lower limbs, which becomes the pathological basis of diabetic foot [22]. The major vascular disease caused by diabetes is mainly atherosclerosis. The main causes may include hyperlipidemia, hyperglycemia, carbon monoxide reduction, insulin resistance, hemagglutination abnormalities, changes in blood flow, etc. [23]. When atherosclerosis occurs in diabetic patients, the plaque contains more calcium, the expression of inflammatory markers increases, and it affects the blood vessels in the more distal part, especially the foot. It is more difficult to form the collateral circulation after vascular occlusion [24]. The risk of diabetic foot ulcer increased significantly after diabetic peripheral arterial disease, and often asymptomatic until the ulcer occurred. Besides, the reduction of blood supply is not only conducive to wound healing and response to infection, but also more likely to make foot lesions [25].

The microvascular complications of diabetes are mainly microcirculatory disorders, including microvascular disease, microvascular disorder, and changes in blood physicochemical properties. In the pathogenesis of diabetic foot, these three interact with each other and cause each other [26]. Diabetes may cause changes in microvascular function, oxygen partial pressure, and vascular permeability, as well as disturbance of rhythmic contraction of arterioles and arterioles. These abnormalities can cause a decrease in capillary blood flow in the skin and an increase in vascular permeability and platelet aggregation, which leads to the thickening of vascular endothelial cells and aggravation of microvascular disease. After a long time, it could cause vascular stenosis, insufficient perfusion of tissues and organs, and blood circulation disorder at the distal end of the limb, which eventually leads to the occurrence of diabetic foot [27].

\section{Diabetic foot caused by infection}

If peripheral neuropathy and vascular lesion are indispensable conditions for the development of the diabetic foot, infection is the direct reason for diabetic foot [28]. The abnormal immune response of diabetic patients leads to the impairment of the phagocytic capacity of neutrophils, which weakens their response to infection or injury. At the same time, ischemia will further aggravate this response [5]. Foot infection generally could be attributed to four independent risk factors: deep trauma, recurrent trauma, long-lasting trauma (>30 days), and 
peripheral vascular disease [29]. About one-third of diabetic patients develop a fever when they have a foot infection, but the number of white blood cells may not increase despite widespread infection. However, if the patient has sensory neuropathy, the perception of pain or temperature is weakened, so the perception of infection will be delayed [30]. If the patient's blood sugar cannot be controlled, the infection will be difficult to control. Also, the blood circulation disorder will eventually lead to the rapid deterioration of the wound [31] (Table 1).

\section{Current therapy for diabetic foot}

Current treatment for diabetic foot includes drug therapy, vascular intervention therapy, dressing adjuvant therapy, hyperbaric oxygen therapy, vacuum suction therapy, growth factor therapy, and stem cell therapy [32].

However, none of the above are sufficient for treating diabetic foot. Drugs used in diabetic foot are mainly for vascular diseases, neuropathy, and hypoglycemic drugs [33]. Drug therapy for patients with mild and moderate diabetic feet simply delays the development of their lesions; it is the basis for the treatment of diabetic foot. Once the drug therapy is not effective and the ischemia is getting serious, the vascular intervention surgery needs to be used to reconstruct the blood flow [34]. However, since most of the diabetics are old and frail, who often suffer from cardiovascular and cerebrovascular diseases, thus, the patients cannot tolerate the stimulation of surgical bypass. Besides, the vascular lesions involve many small arteries and segments, which leads to the lack of distal artery outflow channel in some patients, and thus, the vascular bypass graft and interventional treatment are not applicable [35]. Meanwhile, long-term follow-up showed that patients with diabetic foot were prone to restenosis after vascular intervention. The proportion of patients who opt for amputation remains high [6].

Also, non-surgical therapies include dressing adjuvant therapy, hyperbaric oxygen therapy, and vacuum suction therapy. This kind of simple physical auxiliary intervention could improve the wound inflammation and microcirculation, which promote wound healing, but the treatment requires long-term hospitalization while the effect is not that significant [36]. Cytokine treatment to promote wound healing is a new method for diabetic foot. The cytokines used mainly include fibroblast growth factor (FGF), epidermal growth factor (EGF), platelet growth factor (PGF), vascular endothelial cell growth factor (VEGF), cell colony-stimulating factor(GCSF), and hepatocyte growth factor (HGF) [37].

In recent years, stem cell transplantation has been considering a cutting-edge technology to treat diabetic foot. Although stem cells are not currently used as a routine means for treating diabetic foot in clinical practice, many researchers have reported that stem cell transplantation could promote neovascularization of ischemic limbs, improve and restore limb blood flow, and ultimately cure the diabetic foot [38-41].

\section{Therapy for diabetic foot with stem cells}

Stem cells are a class of cells with self-renewal and multi-directional differentiation potential that can differentiate into specific types of cells under specific conditions [42]. Stem cell transplantation for the treatment of diabetic foot is mainly based on the principle that stem cells can differentiate into vascular endothelial cells and smooth muscle cells in vivo, secreting numerous proangiogenic factors to form new blood vessels. The stem cells are transplanted into the ischemic lower limbs to gradually differentiate and form new capillaries, which improves and restores blood flow to the lower limbs, and achieves the purpose of treating diabetic foot [43].

Currently, the stem cells used to treat diabetic foot are derived from many tissue sources such as the bone marrow, umbilical cord, placenta, cord blood, heart, liver, spleen, pulp, dermis, and adipose tissue. Among them, adipose-derived stem cells are advantageous of the widely distributed but low immunogenicity adipose tissue, which is easier to obtain but less harmful to patients [44]. Many initial studies were adipose-derived stem/ stromal cells (ASCs) identified by Zuk, which have received extensive attention due to their pluripotent differentiation potential, paracrine properties, and significant influence on regenerative medicine [45]. ASCs are a group of homogeneous stem cells obtained by the adherent subculture of freshly isolated SVF. SVF is a

Table 1 Pathology of diabetic foot and the main citation

\begin{tabular}{llll}
\hline Pathology of diabetic foot & & & \\
\hline Neuropathy & Sensory neuropathy & Citations \\
& Motor neuropathy & Foot deformity & [17] [18] \\
& Autonomic neuropathy & Flow-mediated disorder & {$[19]$} \\
Vascular lesion & Macroangiopathy & Atherosclerosis & [21] \\
& Microangiopathy & Microcirculatory disorders & [23] \\
Infection & Main incentive & Accelerate wound deterioration & [28] \\
\hline
\end{tabular}


heterogeneous cell population obtained by digesting adipose tissue [46]. However, compared to ASCs, SVF does not require any cell culture process and can be used directly after separation. Therefore, it is relatively safer and can meet lower regulatory standards, which is favored by many clinical researchers [47].

SVF is widely used to improve diabetic foot [48]. Tan's research found that SVF can increase the viability and migration of fibroblasts in a hyperglycemic environment by upregulating cytokines around the wound, thereby accelerating wound healing [49]. Chae used SVF to treat diabetic foot and found that not only the number of fibroblasts increased significantly, but also the amount of collagen synthesized by fibroblasts increased significantly. The results of subsequent clinical experiments showed that when $100 \%$ of the wounds of patients treated with SVF were healed, the healing rate of the control group was only $62 \%$, indicating that SVF could accelerate wound healing [50] (Table 2).

\section{SVF cells for diabetic foot Acquisition of SVF cells}

The adipose tissue in the human body is generally divided into subcutaneous fat and visceral fat, which can be obtained by liposuction or surgical resection. Common materials include abdomen, buttocks, forearms, groin, etc. [51]. In general, the separation methods of SVF could be generally divided into two categories: enzymatic methods that use proteolytic enzymes to digest adipose tissue and physical and mechanical processing methods that do not use proteolytic enzymes [52]. Enzymatic methods often use type I collagenase to digest adipose tissue. The general process is summarized as follows: the adipose tissue and collagenase are mixed at an appropriate ratio and placed in a $37{ }^{\circ} \mathrm{C}$ environment for $1 \mathrm{~h}$ with shaking and digestion; then an equal amount of complete medium is added to terminate the digestion. The supernatant is discarded by centrifugation, then washed with phosphate buffer saline (PBS), and finally, filtered through a specific pore size filter to obtain an SVF suspension. Physical and mechanical digestion of adipose tissue, including methods such as serum digestion, mechanical shaking, and bolus shearing. Compared to enzymatic methods, this kind of digestion method takes more time, and the cell yield and activity are not good enough; thus, it is not widely used [53].

\section{Cell populations in SVF}

Human adipose tissue can be easily obtained from the abdomen, buttocks, forearm, or groin liposuction or surgical resection. SVF cells could be obtained after adipose tissue is digested with proteolytic enzymes [54]. SVF cells isolated from the adipose tissue are heterogeneous cell populations containing a variety of cells. This multicellular component could be identified by different cell surface molecules. Surface molecules of cells, the cluster of differentiation (CD), could be used to identify different cell types in a cell population [55]. The International Cell Therapy Association has stated to define SVF using this identification method. At present, the academic community has reached a consensus on the cell types contained in SVF cells [56]. SVF cells contain a variety of cell types, such as ASCs; hematopoietic stem cells (HPCs), mesenchymal cells; and endothelial progenitor cells (EPCs), endothelial cells, pericytes, and macrophages [57-59].

\section{Application of SVF in diabetic foot}

SVF cells are able to promote the recovery of the diabetic foot through various effects. SVF, for instance, contains various cells which directly or indirectly secrete bioactive factors to induce degradation of the basement membrane, affecting the proliferation and migration of endothelial cells, thereby promoting the fusion and remodeling of new blood vessels. Also, these cytokines could increase the stability of the pericytes to the vascular network $[13,49,60]$.

\section{Differentiation of SVF}

Among SVF cells, ASCs not only occupy a relatively large amount of cells but also have strong differentiation ability. ASCs are pluripotent stem cells that could differentiate directly into vascular endothelial cells, smooth muscle cells, and pericytes. These cells regulate vascular

Table 2 Diabetic foot treatment and the therapy features

\begin{tabular}{llll}
\hline Treatment of diabetic foot & Features & Citations \\
\hline Therapy & Drug therapy & For mild and moderate patients; just delay the course; basic therapy & \\
& Vascular intervention therapy & Suitable for severe ischemia; except for elderly and weak patients & [33] \\
Dressing adjuvant therapy & Non-surgical therapy; physically assisted intervention; poor effect \\
Hyperbaric oxygen therapy & & \\
Vacuum suction therapy & & Use multiple cytokines; good effect but expensive; new therapy \\
Growth factor therapy & Significant treatment effect; wide range of applications; cutting-edge technology \\
Stem cell therapy & & {$[36]$}
\end{tabular}


Table 3 SVF cells for diabetic foot and its pros and cons

\begin{tabular}{llll}
\hline SVF cells for diabetic foot & & Pros and cons & Citations \\
\hline Separation methods & Enzymatic digestion & Simple and convenient; possible immune response & [52] \\
& Physical method & Safety; long digestion time; low cell yield & [53] \\
Cell populations & Multi-cellular components & Wide source; without culture; safety; effective & [57] \\
Mechanism & Differentiation & Direct action; directly involved in vascular reconstruction & [61] \\
& Paracrine action & Secrete various cytokines; promote vascular remodeling & [66, 69, 71] \\
& Cytokine action & Cytokine interaction; promote cell proliferation and migration & [77] \\
\hline
\end{tabular}

growth, stabilization, and maturation through activation of TGF- $\beta$, angiopoietin-2, PDGF-B/PDGFR- $\beta$, Notch, and S1P/Edg signaling pathways [61, 62]. Meanwhile, pericytes not only promote the appearance of endothelial progenitor cells but also maintain vascular integrity to form a vascular network [63]. Studies have shown that ASCs could also participate in the formation of new micro-vessel together with endothelial cells to form a stable vascular network system [64]. Animal experiments have demonstrated that transplanted ASCs could differentiate into endothelial cells, significantly improving blood flow and capillary density in diabetic and nondiabetic animal models of lower limb ischemia [65].

\section{Paracrine action of SVF}

Studies have found that when SVF cells are transplanted into the ischemic area, their vascular density, blood flow, and secreted hepatocyte growth factor, vascular endothelial growth factor, and basic fibroblast growth factor (bFGF) are significantly increased compared with the control group [66]. If the synthesis of HGF is inhibited, the ability of SVF cells to promote vascularization of ischemic tissue is significantly reduced [67]. Stem cells treated with VEGF antibodies also lose pro-angiogenic capacity in ischemic tissues [68].

ASCs in SVF cells could effectively secrete a large number of pro-angiogenic and anti-apoptotic factors, such as HGF, bFGF, VEGF, PGF-B, and TGF- $\beta$ [69]. Prochzka isolated the factors secreted by ASCs and injected them into the ischemic limbs of rabbits. It was found that the blood perfusion of ischemic tissue in the experimental group was twice as high as that in the control group. Immunohistochemistry showed that the capillary density of the experimental group was significantly higher than the control group, which indicates ASC-secreted cytokines could promote angiogenesis [70].

In addition to ASCs, other components in SVF cells can also promote vascular remodeling through the paracrine pathway. Studies have shown that hypoxia can induce macrophages to secrete vascular regenerative factors such as VEGF and bFGF, thereby promoting the formation of new blood vessels [71]. Macrophages in adipose tissues can be divided into
M1 type macrophages and M2 type macrophages according to their activation states. In SVF cells, more than $90 \%$ of macrophages are M2 type [72]. M2 type macrophage is an anti-inflammatory macrophage. It can secrete anti-inflammatory factors such as IL-4, IL-10, TGF- $\beta$, and pro-angiogenic factors such as bFGF and VEGF, thereby inhibiting the inflammatory response and promoting vascular network formation [73].

Endothelial cells secrete exosomes, and adjacent endothelial cells can act as target cells to bind to exosomes, promoting endothelial growth, migration, and neovascularization [74]. Endothelial cells can activate the ERK1/2 signaling pathway by expressing CXCL-1, induce the secretion of epidermal growth factor, and promote angiogenesis [75]. Besides, stromal cells, fibroblasts, and smooth muscle cells can secrete HGF and regulate angiogenesis [76].

\section{Mechanism of cytokine promoting vascular reconstruction}

SVF cell components or hypoxic tissue can secrete a large amount of cytokines through paracrine, and these active substances can accelerate the healing of diabetic foot by promoting the biological function of the body cells [69-71]. For instance, VEGF could activate endothelial progenitor cells and induce endothelial cells to secrete a variety of cathepsins for degradation of extracellular matrix, as well as inhibit endothelial cell apoptosis and promote endothelial cell proliferation, migration, and neovascularization [77]. HGF binds to its receptor and promotes the proliferation of vascular endothelial cells by activating the Grb2/Sos-Ras-Raf-MAPK signaling pathway [76]. bFGF induces VEGF expression via the FGFR1/c-Src/ p38/NFB-kB signaling pathway [78]. At the same time, the activation of NFB- $\kappa B$ can promote endothelial cell DNA synthesis, cell division, and cell proliferation and promote the regeneration of blood vessels [79]. TGF- $\beta$ contributes to the production of extracellular matrix and promotes the interaction between endothelial cells and parietal cells, which in turn contributes to the formation of blood vessels [80] (Table 3). 


\section{Conclusion and prospect}

As a new therapy for the treatment of diabetic foot, autologous adipose-derived stem cell transplantation has been widely used in clinical practice and has made it possible to exempt some patients from amputation or reduce the amputation plane and improve the quality of life, which fully demonstrates the feasibility of this technology $[15,81]$. SVF is a heterogeneous cell population, which interact with each other to synergize with the whole process of the angiogenesis. However, the specific mechanism of synergistic angiogenesis and reconstruction between components still needs further investigation. Although SVF cells have achieved good therapeutic effects in many studies, further research is still needed to determine whether the cell therapy has side effects such as transplant toxicity and adverse reactions in the longterm treatment. The evaluation of the effect of SVF cells on the treatment of diabetic foot, including the survival of stem cells, the rate of proliferation and transformation, and the determination of the characteristics of transformed cells, still needs to be studied and solved in the future study.

In general, although using SVF cells to treat diabetic foot is still in the early research stage, it has enormous potential in clinical application. It is believed that with further investigation, adipose-derived SVF cells will ultimately show their unique cellular advantages and therapeutic value in clinic.

\section{Abbreviations \\ SVF: Stromal vascular fraction; FGF: Fibroblast growth factor; EGF: Epidermal growth factor; PGF: Platelet growth factor; VEGF: Vascular endothelial cell growth factor; G-CSF: Granulocyte colony-stimulating factor; HGF: Hepatocyte growth factor; ASCs: Adipose-derived stem/stromal cells; PBS: Phosphate buffer saline; CD: Cluster of differentiation; HPCs: Hematopoietic stem cells; EPCs: Endothelial progenitor cells; bFGF: Basic fibroblast growth factor}

\section{Acknowledgements}

Not applicable

\section{Authors' contributions}

$X Z$ was the major contributor in writing the manuscript. WH and LJ reviewed and revised the manuscript. All authors read and approved the final manuscript.

\section{Funding}

There has been no significant financial support for this work that could have influenced its outcome.

\section{Availability of data and materials}

Not applicable

Ethics approval and consent to participate

Not applicable

\section{Consent for publication}

Not applicable

\section{Competing interests}

The authors declare no conflict of interest, financial or otherwise.

\section{Author details}

'School of Life Science and Technology, China Pharmaceutical University, Nanjing 211198, Jiangsu Province, China. ${ }^{2}$ Irell \& Manella Graduate School of Biological Sciences, City of Hope National Medical Center, California 91010, USA. ${ }^{3}$ Department of Endocrinology, The Affiliated ZhongDa Hospital of Southeast University, Nanjing 210009, Jiangsu Province, China.

Received: 28 February 2020 Revised: 15 May 2020

Accepted: 10 July 2020 Published online: 14 September 2020

\section{References}

1. Weck M, Slesaczeck T, Rietzsch $H$, et al. Noninvasive management of the diabetic foot with critical limb ischemia: current options and future perspectives. Ther Adv Endocrinol Metab. 2011;2(6):247-55.

2. Apelqvist J, Bakker $K$, Van Houtum W, et al. International consensus and practical guidelines on the management and the prevention of the diabetic foot. Diabetes Metab Res Rev. 2000;16(S1):84-92.

3. Park JY, Jung HG. Diabetic foot: ulcer, infection, ischemic gangrene. Foot and ankle disorders. Springer. Ther Adv Endocrinol Metab. 2016;24(02):55584.

4. Rahelić D. Of IDF diabetes atlas--call for immediate action. Lijec Vjesn. 2016; 138(1-2):57-8.

5. Shearman CP, Windhaber RJS. Foot complications in patients with diabetes. Surgery (Oxford). 2010;28(6):288-92.

6. Vouillarmet J, Bourron O, Gaudric J, et al. Lower-extremity arterial revascularization: is there any evidence for diabetic foot ulcer-healing? Diabetes Metab. 2016;42(1):4-15.

7. Blumberg $S N$, Berger $A$, Hwang $L$, et al. The role of stem cells in the treatment of diabetic foot ulcers. Diabetes Res Clin Pract. 2012;96(1):1-9.

8. Lopes L, Setia O, Aurshina A, et al. Stem cell therapy for diabetic foot ulcers: a review of preclinical and clinical research. Stem Cell Res Ther. 2018;9(1):188.

9. Zarei F, Negahdari B, Eatemadi A, et al. Diabetic ulcer regeneration: stem cells, biomaterials, growth factors. Artif Cells Nanomed Biotechnol. 2018; 46(1):26-32.

10. Han S, Sun HM, Hwang KC, et al. Adipose-derived stromal vascular fraction cells: update on clinical utility and efficacy. Crit Rev Eukaryot Gene Expr. 2015;25(2):145-52.

11. Zanata F, Shaik S, Devireddy R V, et al. Cryopreserved adipose tissue-derived stromal/stem cells: potential for applications in clinic and therapy. Biobanking and Cryopreservation of Stem Cells. Springer. Adv Exp Med Biol. 2016;951(01):137-46.

12. Moon KC, Chung HY, Han SK, et al. Possibility of injecting adipose-derived stromal vascular fraction cells to accelerate microcirculation in ischemic diabetic feet: a pilot study. Int J Stem Cells. 2019;12(1):107-13.

13. Didangelos $T$, Koliakos $G$, Kouzi K, et al. Accelerated healing of a diabetic foot ulcer using autologous stromal vascular fraction suspended in plateletrich plasma. Regen Med. 2018;13(3):277-81.

14. Lonardi R, Leone N, Gennai S, et al. Autologous micro-fragmented adipose tissue for the treatment of diabetic foot minor amputations: a randomized controlled single-center clinical trial (MiFrAADiF). Stem Cell Res Ther. 2019; 10(1):223.

15. Andia I, Maffulli N, Burgos AN. Stromal vascular fraction technologies and clinical applications. Expert Opin Biol Ther. 2019;19(12):1289-05.

16. Pafili K, Papanas N, Ziegler D. Neuropathy in diabetes:"one cannot begin it too soon". Angiology. 2018;69(9):752-4.

17. Jaiswal M, Divers J, Urbina $E_{1}$ et al. Cardiovascular autonomic neuropathy in adolescents and young adults with type 1 and type 2 diabetes: the SEARCH for diabetes in youth cohort study. Pediatr Diabetes. 2018;19(4):680-9.

18. Kalish J, Hamdan A. Management of diabetic foot problems. Int J Surg. 2010:51(2):476-86.

19. Papanas N, Maltezos E. The diabetic foot: a global threat and a huge challenge for Greece. Hippokratia. 2009;13(4):199-04.

20. Vinik Al, Casellini C, Parson HK, et al. Cardiac autonomic neuropathy in diabetes: a predictor of cardiometabolic events. Front Neurosci. 2018;12:591.

21. Andrews KL, Dyck PJ, Kavros SJ, et al. Plantar ulcers and neuropathic arthropathies: associated diseases, polyneuropathy correlates, and risk covariates. Adv Skin Wound Care. 2019:32(4):168-75.

22. Troisi N, Ercolini L, Chisci E, et al. Diabetic foot infection: preliminary results of a fast-track program with early endovascular revascularization and local surgical treatment. Ann Vasc Surg. 2016;30:286-91. 
23. Green $\mathrm{E}$, Johnson B. Evaluation and management of vascular disease in the diabetic patient. The Surgical Management of the Diabetic Foot and Ankle. Springer. 2016. p. 19-27. https://doi.org/10.1007/978-3-319-27623-6_3.

24. Ferik S, Güven H, Ateş MP, et al. Diabetic polyneuropathy, deep white matter lesions, and carotid atherosclerosis: is there any association? Neurol Sci. 2018;39(1):103-10.

25. Malyar NM, Freisinger E, Meyborg M, et al. Amputations and mortality in inhospital treated patients with peripheral artery disease and diabetic foot syndrome. J Diabetes Complicat. 2016;30(6):1117-22.

26. Dioszegi $A$, Vass $M$, Nemeth $N$, et al. Rheopheresis treatment of diabetic foot syndrome. Atherosclerosis. 2017;263:e272.

27. American Diabetes Association. Microvascular complications and foot care: standards of medical care in diabetes 2018. Diabetes Care. 2018;41(1):S105-18.

28. Lavery LA, Ryan EC, Ahn J, Crisologo PA, Oz OK, La Fontaine J, Wukich DK. The Infected Diabetic Foot: Reevaluating the Infectious Diseases Society of America Diabetic Foot Infection Classification. Clin Infect Dis. 2020;70(8): 1573-9. https://doi.org/10.1093/cid/ciz489.

29. Lavery LA, Armstrong DG, Wunderlich RP, et al. Risk factors for foot infections in individuals with diabetes. Diabetes Care. 2006;29(6):1288-93.

30. Markakis K, Faris A, Sharaf H, et al. Local antibiotic delivery systems: current and future applications for diabetic foot infections. Int J Low Extrem Wounds. 2018;17(1):14-21.

31. Eltzschig H, Eckle T. Ischemia and reperfusion—from mechanism to translation. Nat Med. 2011;17(11):1391-01.

32. Lipsky BA. Diabetic foot infections: current treatment and delaying the post antibiotic era. Diabetes Metab Res Rev. 2016;32:246-53.

33. Karri V, Kuppusamy G, Talluri SV, et al. Current and emerging therapies in the management of diabetic foot ulcers. Curr Med Res Opin. 2016;32(3):519-42.

34. Ibrahim A, Practice C. IDF clinical practice recommendation on the diabetic foot: a guide for healthcare professionals. Diabetes Res Clin Pract. 2017;127: 285-7.

35. Joret M, Dean A, Cao C, et al. The financial burden of surgical and endovascular treatment of diabetic foot wounds. J Vasc Surg. 2016;64(3): 648-55.

36. Mutluoglu M, Lipsky B. Non-surgical treatment of diabetic foot osteomyelitis. Lancet Diabetes Endocrinol. 2017:5(8):668.

37. Rivero-González A, Martín-Izquierdo E, Marín-Delgado C, et al. Cytokines in diabetes and diabetic complications. Cytokine Effector Functions in Tissues. Elsevier. Academic Press; 2017. p. 119-28. https://doi.org/10.1016/B978-0-12804214-4.00006-3.

38. Cao Y, Gang X, Sun C, et al. Mesenchymal stem cells improve healing of diabetic foot ulcer. J Diabetes Res. 2017;2017:9328-47.

39. Mizukami H, Yagihashi S. Is stem cell transplantation ready for prime time in diabetic polyneuropathy? Curr Diab Rep. 2016;16(9):86.

40. Qin H, Zhu X, Zhang B, et al. Clinical evaluation of human umbilical cord mesenchymal stem cell transplantation after angioplasty for diabetic foot. Exp Clin Endocrinol Diabetes. 2016;124(08):497-03.

41. Wu Q, Lei $X, C$ hen $L$, et al. Autologous platelet-rich gel combined with in vitro amplification of bone marrow mesenchymal stem cell transplantation to treat the diabetic foot ulcer: a case report. Ann Transl Med. 2018;6(15):307.

42. Gehling UM, Ergün S, Schumacher U, et al. In vitro differentiation of endothelial cells from AC133-positive progenitor cells. Blood. 2000;95(10):3106-12.

43. Tateishi-Yuyama E, Matsubara H, Murohara T, et al. Therapeutic angiogenesis for patients with limb ischaemia by autologous transplantation of bonemarrow cells: a pilot study and a randomised controlled trial. Lancet. 2002; 360(9331):427-35.

44. Tobita M, Orbay $H$, Mizuno $H$. Adipose-derived stem cells: current findings and future perspectives. Discov Med. 2011;11(57):160-70.

45. Zuk PA, Zhu M, Mizuno $H$, et al. Multilineage cells from human adipose tissue: implications for cell-based therapies. Tissue Eng. 2001;7(2):211-28

46. Locke M, Windsor J, Dunbar P. Human adipose-derived stem cells: isolation, characterization and applications in surgery. ANZ J Surg. 2009;79(4):235-44.

47. Domergue $\mathrm{S}$, Bony $\mathrm{C}$, Maumus $\mathrm{M}$, et al. Comparison between stromal vascular fraction and adipose mesenchymal stem cells in remodeling hypertrophic scars. PLoS One. 2016;11(5):e0156161.

48. Haney N, Gabrielson A, Kohn T, et al. The use of stromal vascular fraction in the treatment of male sexual dysfunction: a review of preclinical and clinical studies. Sex Med Rev. 2019;7(2):313-20.

49. Tan S, Yeo $X$, Liang Z, et al. Stromal vascular fraction promotes fibroblast migration and cellular viability in a hyperglycemic microenvironment through up-regulation of wound healing cytokines. Exp Mol Pathol. 2018; 104(3):250-5.

50. Chae DS, Han S, Son M, et al. Stromal vascular fraction shows robust wound healing through high chemotactic and epithelialization property. Cytotherapy. 2017;19(4):543-54.

51. Piché ME, Vasan SK, Hodson L, et al. Relevance of human fat distribution on lipid and lipoprotein metabolism and cardiovascular disease risk. Curr Opin Lipidol. 2018;29(4):285-92.

52. Shani N, Gur E, Solodeev I, et al. Mechanical apparatus and method for isolating stromal vascular fraction: U.S. Patent Application. 2018;15:579-02.

53. Van D, Tuin A, Spiekman M, et al. Comparison of intraoperative procedures for isolation of clinical grade stromal vascular fraction for regenerative purposes: a systematic review. J Tissue Eng Regen Med. 2018;12(1):e261-74.

54. Bourin P, Bunnell BA, Casteilla $L$, et al. Stromal cells from the adipose tissuederived stromal vascular fraction and culture expanded adipose tissuederived stromal/stem cells: a joint statement of the International Federation for Adipose Therapeutics and Science (IFATS) and the International Society for Cellular Therapy (ISCT). Cytotherapy. 2013;15(6):641-8.

55. Michelle L, John W, Rod D. Human adipose-derived stem cells: isolation, characterization and applications in surgery. ANZ J Surg. 2010;79(4):235-44.

56. Nguyen A, Guo J, Banyard D, et al. Stromal vascular fraction: a regenerative reality? Part 1: current concepts and review of the literature. J Plast Reconstr Aesthet Surg. 2016;69(2):170-9.

57. Guo J, Nguyen A, Banyard D, et al. Stromal vascular fraction: a regenerative reality? Part 2: mechanisms of regenerative action. J Plast Reconstr Aesthet Surg. 2016;69(2):180-8.

58. Bora P, Majumdar AS. Adipose tissue-derived stromal vascular fraction in regenerative medicine: a brief review on biology and translation. Stem Cell Res Ther. 2017;8(1):145.

59. Gentile P, Orlandi A, Scioli MG, et al. Concise review: adipose-derived stromal vascular fraction cells and platelet-rich plasma: basic and clinical implications for tissue engineering therapies in regenerative surgery. Stem Cells Transl Med. 2012;1(3):230-6.

60. Gadelkarim M, Abushouk Al, Ghanem E, et al. Adipose-derived stem cells: effectiveness and advances in delivery in diabetic wound healing. Biomed Pharmacother. 2018;107:625-33.

61. Maumus M, Peyrafitte J, D'angelo R, et al. Native human adipose stromal cells: localization, morphology and phenotype. Int J Obes. 2011;35(9):1141-53.

62. Gimble J, Katz A, Bunnell B. Adipose-derived stem cells for regenerative medicine. Circ Res. 2007;100(9):1249-60.

63. Mills J, Cowin AJ, Kaur PJC. Pericytes, mesenchymal stem cells and the wound healing process. Cells. 2013;2(3):621-34.

64. Paquet J, Moya A, Bensidhoum M, et al. Engineered cell-free scaffold with twostage delivery of miRNA-26a for bone repair. Ann Transl Med. 2016;4(10):204.

65. Miranville A, Heeschen C, Sengenes C, et al. Improvement of postnatal neovascularization by human adipose tissue-derived stem cells. Circulation. 2004;110(3):349-55.

66. Atalay S, Coruh A, Deniz KJ. Stromal vascular fraction improves deep partial thickness burn wound healing. Burns. 2014;40(7):1375-83.

67. Cai L, Johnstone BH, Cook TG, et al. Suppression of hepatocyte growth factor production impairs the ability of adipose-derived stem cells to promote ischemic tissue revascularization. Stem Cells. 2007;25(12):3234-43.

68. Sasaki Kl, Murohara $\mathrm{T}$, Ikeda $\mathrm{H}$, et al. Evidence for the importance of angiotensin II type 1 receptor in ischemia-induced angiogenesis. J Clin Invest. 2002;109(5):603-11.

69. Kwon HM, Hur SM, Park KY, et al. Multiple paracrine factors secreted by mesenchymal stem cells contribute to angiogenesis. Vasc Pharmacol. 2014; 63(1):19-28

70. Procházka V, Jurčíková J, Laššák O, et al. Therapeutic potential of adiposederived therapeutic factor concentrate for treating critical limb ischemia. Cell Transplant. 2016;25(9):1623-33.

71. Nucera S, Biziato D, Palma M. The interplay between macrophages and angiogenesis in development, tissue injury and regeneration. Int J Dev Biol. 2011;55(4-5):495-03.

72. Eto $H$, Ishimine $H$, Kinoshita $K$, et al. Characterization of human adipose tissue-resident hematopoietic cell populations reveals a novel macrophage subpopulation with CD34 expression and mesenchymal multipotency. Stem Cells Dev. 2012;22(6):985-97.

73. Li P, Lu M, Nguyen M, et al. Functional heterogeneity of CD11c-positive adipose tissue macrophages in diet-induced obese mice. J Biol Chem. 2010; 285(20):15333-45. 
74. Van B, De J, Smits M, et al. Endothelial cells require miR-214 to secrete exosomes that suppress senescence and induce angiogenesis in human and mouse endothelial cells. Blood. 2013;121(19):3997-06.

75. Miyake M, Goodison S, Urquidi V, et al. Expression of CXCL1 in human endothelial cells induces angiogenesis through the CXCR2 receptor and the ERK1/2 and EGF pathways. Lab Investig. 2013;93(7):768-78.

76. Newman A, Chou W, Welch RK, et al. Analysis of stromal cell secretomes reveals a critical role for stromal cell-derived hepatocyte growth factor and fibronectin in angiogenesis. Arterioscler Thromb Vasc Biol. 2013;33(3):513-22.

77. Hao C, Shintani S, Shimizu Y, et al. Therapeutic angiogenesis by autologous adipose-derived regenerative cells: comparison with bone marrow mononuclear cells. Am J Physiol Heart Circ Physiol. 2014;307(6):H869-H79.

78. Tzeng $\mathrm{H}$, Chen $\mathrm{P}$, Lin $\mathrm{K}$, et al. Basic fibroblast growth factor induces VEGF expression in chondrosarcoma cells and subsequently promotes endothelial progenitor cell-primed angiogenesis. Clin Sci (Lond). 2015;129(2):147-58.

79. Yi E, Kim Y. Xylitol inhibits in vitro and in vivo angiogenesis by suppressing the NF-KB and Akt signaling pathways. Int J Oncol. 2013:43(1):315-20.

80. Hirschi K, Skalak T, Peirce SM, et al. Vascular assembly in natural and engineered tissues. Ann N Y Acad Sci. 2002;961(1):223-42.

81. Eyuboglu A, Uysal C, Ozgun G, et al. The effect of adipose derived stromal vascular fraction on stasis zone in an experimental burn model. Burns. 2018; 44(2):386-96.

\section{Publisher's Note}

Springer Nature remains neutral with regard to jurisdictional claims in published maps and institutional affiliations. 